\title{
Substrate Shift Reveals Roles for Members of Bacterial Consortia in Degradation of Plant Cell Wall Polymers
}

\author{
Camila Carlos ${ }^{1,2 *}$, Huan Fan ${ }^{1}$ and Cameron R. Currie ${ }^{1,2 *}$ \\ ${ }^{1}$ Department of Bacteriology, University of Wisconsin-Madison, Madison, WI, United States, ${ }^{2}$ U.S. Department of Energy, \\ Great Lakes Bioenergy Research Center, University of Wisconsin-Madison, Madison, WI, United States
}

Deconstructing the intricate matrix of cellulose, hemicellulose, and lignin poses a major challenge in biofuel production. In diverse environments in nature, some microbial communities, are able to overcome plant biomass recalcitrance. Identifying key degraders of each component of plant cell wall can help improve biological degradation of plant feedstock. Here, we sequenced the metagenome of lignocellulose-adapted

OPEN ACCESS

Edited by:

Graeme W. Nicol,

Université de Lyon, France

Reviewed by:

Manuel Kleiner,

North Carolina State University,

United States

Patrick K. H. Lee,

City University of Hong Kong,

Hong Kong

*Correspondence:

Camila Carlos

camilacarlos@gmail.com

Cameron R. Currie

currie@bact.wisc.edu

Specialty section:

This article was submitted to

Terrestrial Microbiology,

a section of the journal

Frontiers in Microbiology

Received: 19 September 2017

Accepted: 15 February 2018

Published: 01 March 2018

Citation:

Carlos C, Fan $\mathrm{H}$ and Currie CR (2018) Substrate Shift Reveals Roles for Members of Bacterial Consortia

in Degradation of Plant Cell Wall

Polymers. Front. Microbiol. 9:364.

doi: 10.3389/fmicb.2018.00364 microbial consortia sub-cultured on xylan and alkali lignin media. We observed a drastic shift on community composition after sub-culturing, independently of the original consortia. Proteobacteria relative abundance increased after growth in alkali lignin medium, while Bacteroidetes abundance increased after growth in xylan medium. At the genus level, Pseudomonas was more abundant in the communities growing on alkali lignin, Sphingobacterium in the communities growing on xylan and Cellulomonas abundance was the highest in the original microbial consortia. We also observed functional convergence of microbial communities after incubation in alkali lignin, due to an enrichment of genes involved in benzoate degradation and catechol orthocleavage pathways. Our results represent an important step toward the elucidation of key members of microbial communities on lignocellulose degradation and may aide the design of novel lignocellulolytic microbial consortia that are able to efficiently degrade plant cell wall polymers.

Keywords: microbial enrichment, lignocellulose, xylan, lignin, Pseudomonas, Sphingobacterium

\section{INTRODUCTION}

Plant biomass is renewable and rich in energy, and as such, is a promising fuel alternative to fossil fuels (Goldemberg, 2009; Liao et al., 2016). However, low efficient plant cell wall deconstruction is a major barrier for economically viable biofuel production (Klein-Marcuschamer and Blanch, 2015). Plant cell walls are composed of a matrix of heteropolymers (i.e., cellulose, hemicellulose, and lignin) that are physically structured to prevent enzymatic breakdown. Commonly, a chemical and/or physical pretreatment of lignocellulosic material is applied to make cellulose accessible to hydrolytic enzymes through the solubilization of hemicelluloses and/or lignin (Mosier et al., 2005).

Natural or engineered microbial consortia are a promising approach to overcoming plant biomass recalcitrance, because of their ability to extract the majority of the energy from 
plant polymers in nature (Wei et al., 2009; Gessner et al., 2010; Lewin et al., 2016). The enzymatic degradation of plant material depends on multiple biomass-degrading enzymes, with complementary and synergistic activities, which are secreted by various species of micro-organisms (Allgaier et al., 2010; DeAngelis et al., 2010; Cragg et al., 2015; Lemos et al., 2017). Microbial enrichment in specific lignocellulose material is a common approach to obtain efficient and simple microbial assemblages that are able to degrade plant cell wall components (de Lima Brossi et al., 2016; Jiménez et al., 2016; Korenblum et al., 2016; Maruthamuthu et al., 2016; Mello et al., 2016). Cellulose-degrading consortia have been obtained from leafcutter ant refuse dumps (Lewin et al., 2016), and mixtures of plant material and chicken feces (Haruta et al., 2002). Lignindegrading consortia have been obtained from wetland (Wang et al., 2013), rice paddy field soil (Kato et al., 2015), and redwood compost (Ceballos et al., 2017). When compared to the environmental inoculum, the enrichment derived consortia show reduced diversity, as a result of the limited number of ecological niches and selection toward more efficient degraders (Jiménez et al., 2017). The taxonomic and functional composition of lignocellulolytic microbial consortia are mainly influenced by the community in the environmental inoculum, and the substrate used for the enrichment (Cortes-Tolalpa et al., 2016; de Lima Brossi et al., 2016; Wong et al., 2016). For example, Wong et al. (2016) compared microbial enrichments derived from beaver dropping and moose rumen and found that the substrate is a major driver of the microbial consortia composition. Jiménez et al. (2016) used forest soil as an inoculum and also found distinct taxonomic composition in bacterial communities enriched in wheat straw, switchgrass and corn stover, but with similar profiles of carbohydrateactive enzyme families. Interestingly, in these studies, even after several batch cultures, the resulting microbial consortia still presented dozens of bacterial species. In plant biomass degradation, coexistence of several microbial species is possible as different members of a consortium occupy different niches, i.e., have different carbon source preferences (Jiménez et al., 2017).

In this study, we aimed to identify members of microbial consortia that have specific preferences for two major components of plant cell wall, xylan and lignin. Eight plant biomass-degrading microbial consortia were subcultured in minimal medium with xylan or alkali lignin as the sole carbon source. Deep metagenomics sequencing of these communities revealed changes in the abundance of distinct bacterial members in response to xylan and lignin-derived compounds. Furthermore, we identified metabolic pathways that were enriched after the substrate shifting.

\section{MATERIALS AND METHODS}

\section{Enrichment Cultures}

Three chicken feces and five soil samples were aseptically collected in DeForest, WI (N 43.204365, W -89.283446) in September 28,2015 and stored at $4^{\circ} \mathrm{C}$ for 1 day until inoculation
(Table 1). Samples were collected around $5 \mathrm{~m}$ from each other. Each sample was homogenized with sterile phosphate-buffered saline (PBS) and $10 \mu \mathrm{L}$ of the solution was inoculated in $5 \mathrm{~mL}$ of autoclaved minimal medium with $0.025 \mathrm{~g}$ of poplar wood chips (size $<1 \mathrm{~mm}$ ) or $1 \mathrm{~cm} \times 10 \mathrm{~cm}$ strip of Whatman \#1 filter paper. M63 minimal medium was used, containing $61.5 \mathrm{mM}$ potassium phosphate, $38.5 \mathrm{mM}$ potassium phosphate monobasic, $15.1 \mathrm{mM}$ ammonium sulfate, $0.5 \mathrm{mg} / \mathrm{L}$ of iron sulfate, $1 \mathrm{mM}$ magnesium sulfate solution, $1 \mathrm{mg} / \mathrm{L}$ of thiamine, and $5 \mathrm{~mL} / \mathrm{L}$ of SPV-4 trace elements solution (Voelskow, 1988). Consortia were grown at $30^{\circ} \mathrm{C}$ at $250 \mathrm{rpm}$. After 7 days samples were vortexed, and $50 \mu \mathrm{L}$ from each was transferred into tubes containing $5 \mathrm{~mL}$ of sterile M63 medium with one enrichment substrate (filter paper or wood chips). After 24 passages, these consortia were centrifuged at $12,000 \mathrm{~g}$ for $5 \mathrm{~min}$ and the pellets were stored at $-80^{\circ} \mathrm{C}$ until DNA extraction. Two microliter of these consortia were inoculated to $3 \mathrm{~mL}$ of autoclaved M63 medium containing $5 \%(\mathrm{w} / \mathrm{v})$ of beechwood xylan (Megazyme) or alkali lignin with low sulfonate content (Sigma-Aldrich) and incubated at $30^{\circ} \mathrm{C}$ at $250 \mathrm{rpm}$ for 7 days. Cultures were centrifuged at 12,000 $\mathrm{g}$ for $5 \mathrm{~min}$ and the pellets were maintained at $-80^{\circ} \mathrm{C}$ until DNA extraction.

\section{Metagenome DNA Extraction Sequencing, Assembly, and Annotation}

Metagenomic DNA was extracted using the PowerSoil ${ }^{\circledR}$ DNA Isolation Kit (Mo Bio, Carlsbad, CA, United States) following the manufacturer instructions. The DNA was quantified using a Qubit Kit (Invitrogen, Carlsbad, CA, United States), and the integrity was confirmed by $1 \%$ agarose gel electrophoresis. DNA yields are shown in Supplementary Table S1. Metagenomic library construction and sequencing were carried out at the Joint Genome Institute ${ }^{1}$ using the Illumina HiSeq-2500 platform $(2 \times 150 \mathrm{bp})$. Read ends were trimmed to a minimum quality score of 12 and filtered to have a maximum of three ambiguous bases and a minimum length of 51 . Trimmed and filtered pairedend reads were assembled using the Megahit assembler ( $\mathrm{Li}$ D. et al., 2016) with the default setting except the following option:“-k-list 23,43,63,83,103,123." Functional annotation and taxonomic classification were performed using the Integrated Microbial Genomes pipeline (Chen et al., 2016; Huntemann et al., 2016). The assembled metagenomes were deposited in the NCBI whole genome sequence (WGS) as BioProject PRJNA422409.

\section{Statistical and Bioinformatics Analyses}

KEGG Orthologous (KO) distributions were normalized as counts per million reads (CPMR) calculated as $(R / N)^{*} 10^{6}$, where $R$ is the number of mapped reads to a gene and $N$ is the total number of reads of that sample. One-way analysis of similarity (ANOSIM) and principal coordinates analysis (PCoA), using Morisita index, were performed with PAST software version $3.15^{2}$. The significance of the relative abundance difference in $\mathrm{KO}$ was performed using Kruskal-Wallis and multiple test correction via Benjamini and Hochberg false discovery rate, which are

\footnotetext{
${ }^{1}$ http://www.jgi.doe.gov

${ }^{2}$ http://folk.uio.no/ohammer/past/
} 
TABLE 1 | Description of the metagenomic samples used in this study.

\begin{tabular}{|c|c|c|c|c|c|c|c|c|}
\hline $\begin{array}{l}\text { Sample } \\
\text { name }\end{array}$ & Treatment & Inoculum & $\begin{array}{l}\text { Enrichment } \\
\text { substrate }\end{array}$ & $\begin{array}{c}\text { Total number } \\
\text { of reads } \\
\text { passed QC }\end{array}$ & $\begin{array}{l}\text { Total number } \\
\text { of bases in } \\
\text { contigs }\end{array}$ & $\begin{array}{c}\text { Total gene } \\
\text { count }\end{array}$ & IMG ID & IMG Name \\
\hline S1 & Original & Soil & Filter paper & $14,071,782$ & $70,033,963$ & 95,832 & 3300012821 & DID2877_E24 \\
\hline S1.Lig & Lignin & Soil & Filter paper & $13,863,076$ & $61,021,420$ & 80,653 & 3300012804 & DID2877_E24_Lignin \\
\hline S1.Xyl & Xylan & Soil & Filter paper & $13,206,130$ & $66,984,622$ & 80,990 & 3300012811 & DID2877_E24_Xylan \\
\hline S2 & Original & Soil & Wood chips & $15,279,458$ & $78,306,800$ & 112,814 & 3300012799 & DID2878_E24 \\
\hline S2.Lig & Lignin & Soil & Wood chips & $16,388,664$ & $80,882,717$ & 107,226 & 3300012801 & DID2878_E24_Lignin \\
\hline S2.Xyl & Xylan & Soil & Wood chips & $17,105,142$ & $54,474,056$ & 77,518 & 3300012796 & DID2878_E24_Xylan \\
\hline $\mathrm{C} 3$ & Original & Chicken feces & Wood chips & $14,985,520$ & $152,610,958$ & 224,072 & 3300012862 & DID2937_E24 \\
\hline C3.Lig & Lignin & Chicken feces & Wood chips & $15,514,838$ & $102,584,925$ & 137,408 & 3300012840 & DID2937_E24_Lignin \\
\hline C3.Xyl & Xylan & Chicken feces & Wood chips & $16,445,174$ & $226,810,859$ & 337,114 & 3300012879 & DID2937_E24_Xylan \\
\hline C1 & Original & Chicken feces & Filter paper & $14,249,310$ & $87,284,368$ & 112,117 & 3300012810 & DID2882_E24 \\
\hline C1.Lig & Lignin & Chicken feces & Filter paper & $13,511,478$ & $63,445,060$ & 87,862 & 3300012800 & DID2882_E24_Lignin \\
\hline C1.Xyl & Xylan & Chicken feces & Filter paper & $12,633,900$ & $72,633,020$ & 96,433 & 3300012802 & DID2882_E24_Xylan \\
\hline $\mathrm{C} 2$ & Original & Chicken feces & Filter paper & $16,753,258$ & $47,006,209$ & 57,079 & 3300012823 & DID2898_E24 \\
\hline C2.Lig & Lignin & Chicken feces & Filter paper & $15,495,898$ & $57,746,371$ & 80,856 & 3300012817 & DID2898_E24_Lignin \\
\hline C2.Xyl & Xylan & Chicken feces & Filter paper & $16,400,218$ & $48,127,918$ & 70,168 & 3300012797 & DID2898_E24_Xylan \\
\hline S3 & Original & Soil & Wood chips & $16,445,174$ & $7,592,135$ & 9,196 & 3300012789 & DID2925_E24 \\
\hline S3.Lig & Lignin & Soil & Wood chips & $7,817,840$ & $10,282,172$ & 15,310 & 3300012792 & DID2925_E24_Lignin \\
\hline S3.Xyl & Xylan & Soil & Wood chips & 8,087,312 & $10,926,255$ & 12,253 & 3300012791 & DID2925_E24_Xylan \\
\hline S4 & Original & Soil & Wood chips & $16,388,570$ & $108,195,268$ & 141,668 & 3300012842 & DID2933_E24 \\
\hline S4.Lig & Lignin & Soil & Wood chips & $23,490,534$ & $92,910,293$ & 128,456 & 3300012833 & DID2933_E24_Lignin \\
\hline S4.Xyl & Xylan & Soil & Wood chips & $13,402,340$ & $70,611,347$ & 89,703 & 3300012808 & DID2933_E24_Xylan \\
\hline S5 & Original & Soil & Wood chips & $13,830,936$ & $135,709,802$ & 199,758 & 3300012859 & DID2934_E24 \\
\hline S5.Lig & Lignin & Soil & Wood chips & $9,743,042$ & $67,969,669$ & 112,711 & 3300012822 & DID2934_E24_Lignin \\
\hline S5.Xyl & Xylan & Soil & Wood chips & $15,354,958$ & $90,451,188$ & 125,640 & 3300012830 & DID2934_E24_Xylan \\
\hline
\end{tabular}

implemented in the STAMP software version 2.1.3 $3^{3}$. Relative abundance of bacterial genera in each sample was obtained by dividing the number of reads mapped to all the contigs classified to a genus by the total number of reads in each sample. ANOVA was used to test whether there are significant differences between the relative abundance of bacterial genera from those consortia. Contigs were assigned to the last common ancestor where at least $30 \%$ of the genes had USEARCH hits (Huntemann et al., 2016). Only contigs with 10 or more genes were used for this analysis. A network of association between bacterial genera and samples was visualized using Cytoscape v3.0 software.

\section{Cluster Analysis Based on $\boldsymbol{k}$-mer Composition of Each Sample}

In order to understand the relative similarities among the metagenomic datasets, we looked at the $k$-mer (short DNA strands with $k$ base pairs) composition of each sample. We fed the contigs into a $k$-mer composition analysis pipeline, the AAF package (Fan et al., 2015). The distances used for clustering by the AAF package are the dissimilarities derived from the proportion of unique $k$-mers that are shared between each pair of samples. The parameters we used were $k=23$ (the length of the $k$-mers) and $n=1$ (minimum frequency of each $k$-mer to be considered present). $k$ should be long enough to overcome the problem of

${ }^{3}$ http://kiwi.cs.dal.ca/Software/STAMP homoplasy. We tested different $k$ until the topology stabilized at 23. The minimum frequency is designed to filter out $k$-mers calculated from raw reads containing sequencing errors, which are usually less frequent than average sequencing depth. Because $k$-mers were calculated from assemblies in our case, $n$ is set to 1 , therefore no $k$-mer was filtered out.

We also looked at the Average Nucleotide Identity (ANI) between the assemblies of different metagenomic datasets, and used the dissimilarities (1-ANI) for the same type clustering analysis. ANI was calculated using pyani (Pritchard et al., 2016) with MUMmer (Kurtz et al., 2004) as the sequence aligner.

\section{RESULTS}

\section{Taxonomic Affiliation of Contigs and Single-Copy Genes}

The 24 microbial consortia (eight original enrichments, eight subcultures in xylan, and eight subcultures in alkali lignin) were sequenced and a total of $52 \mathrm{~Gb}$ of high quality reads were obtained (Table 1). To identify the most abundant microorganisms growing on each carbon source, we used coverage information associated with the contigs classified by IMG to genus level (Markowitz et al., 2013). Figure 1A shows the relative abundance of each genus for the 20 most abundant genera found across the microbial consortia. In 

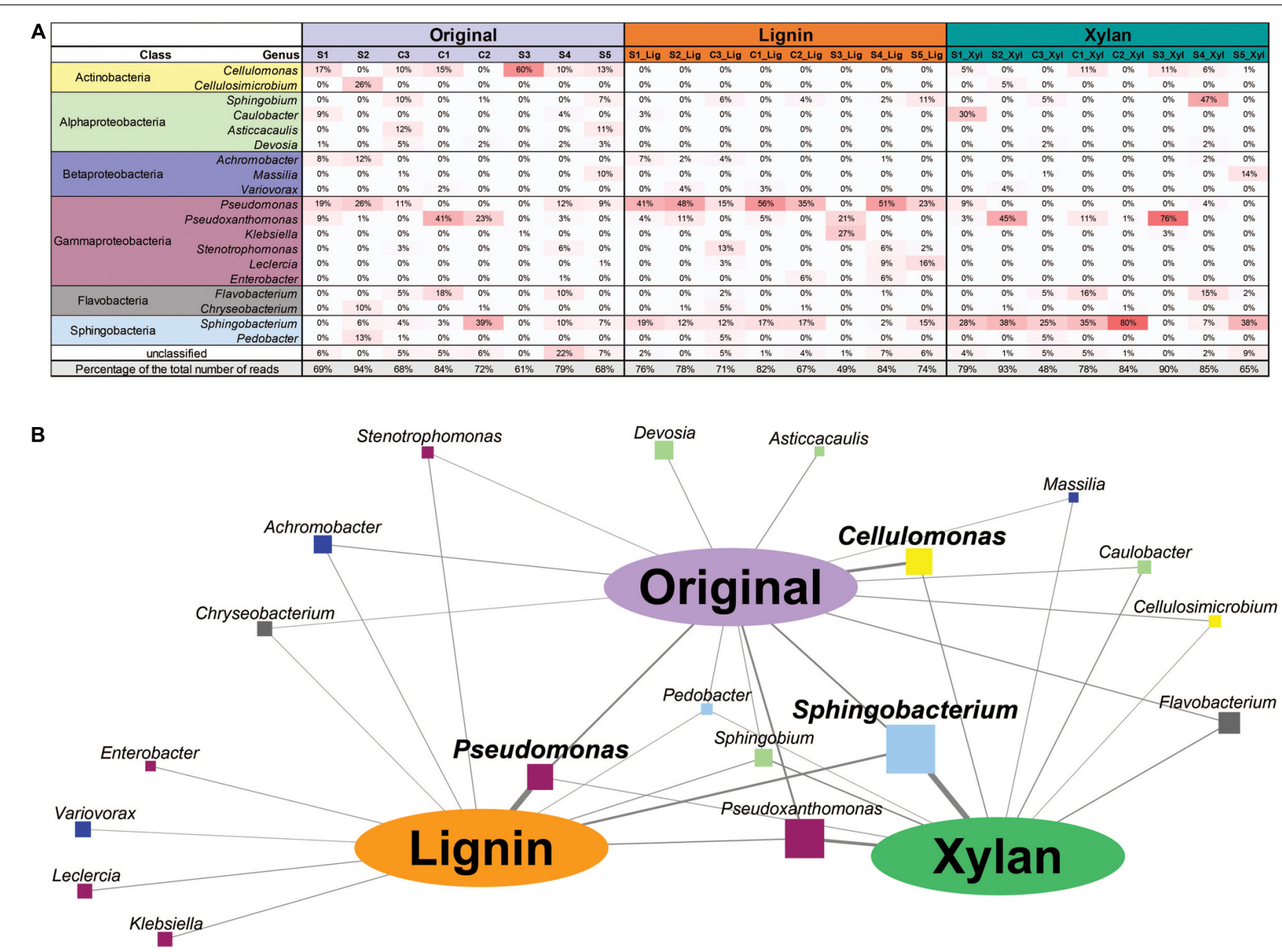

FIGURE 1 | (A) Heatmap showing the relative abundance of the top bacterial genera assigned to contigs with 10 or more genes. (B) Association network between bacterial genera and carbon sources. Oval nodes represent carbon sources used in this study; squares nodes represent the top bacterial genera assigned to contigs with 10 or more genes; the size of the square node is proportional to the overall relative abundance of the bacterial genera across all the samples. Edge width represents the average relative abundance of the bacterial genera in each carbon source.

average, the relative abundance of Pseudomonas was higher after growth in alkali lignin medium than in the original consortia (ANOVA, $F=9.893, p=0.007$ ). Consortium S3_Lig showed an increase in the abundance of Klebsiella after growth in alkali lignin medium. Cellulomonas was not detected in any of the communities grown in alkali lignin medium and on average, it was not significantly different between xylan substrate and the original consortia. On average, Sphingobacterium abundance increased after growth in xylan medium when compared to the original consortia (ANOVA, $F=5.659, p=0.032$ ). Pseudoxanthomonas relative abundance increased after growth in xylan in consortium S3_Xyl, and Sphingobium increased in consortium S4_Xyl.

To further explore the association between bacterial genera and carbon source, a network was built, where the width of the edges represents the average abundance of each genus in each treatment (Figure 1B). The strongest associations were among Sphingobacterium and Pseudoxanthomonas with xylan, Pseudomonas with alkali lignin, and Cellulomonas with the original communities. Some genera were only associated with alkali lignin, such as Klebsiella, Variovorax, Leclercia, and Enterobacter and no genus was exclusively associated with xylan.

Community diversity was assessed via two single-copy genes (alanyl-tRNA synthetase: COG0013 and excinuclease ABC subunit B UvrB: COG0556) revealed similar taxonomic patterns (Supplementary Table S2). The diversity of COG0013 phylotypes varied from two to 19 per sample and the COG0556 varied from two to 17 phylotypes per sample. For both phylomarkers, no significant change in the number of phylotypes was found among the treatments.

\section{Microbial Community Shifts}

Using $k$-mer composition, the microbial communities were clustered according to the enrichment community line (Figure 2A). The same trend is shown with the clustering based on ANI (Supplementary Figure S1). While their KO functional profiles revealed that the microbial communities are 

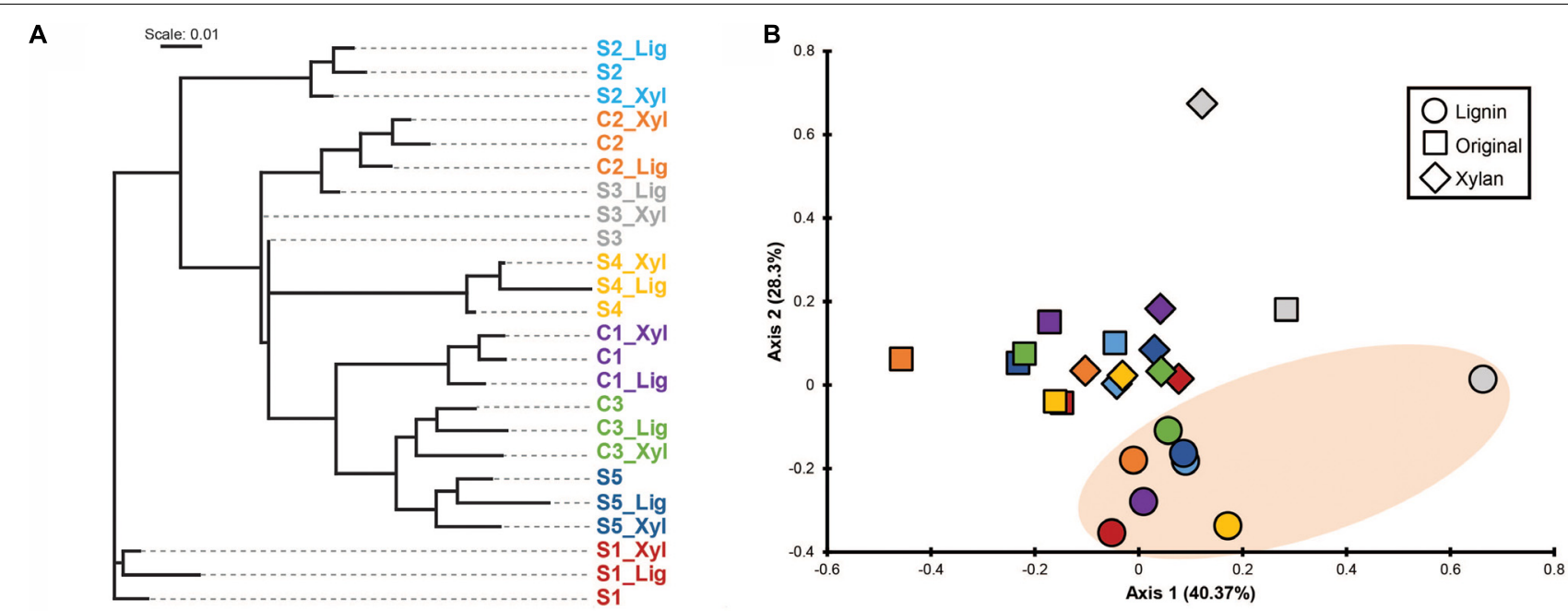

C
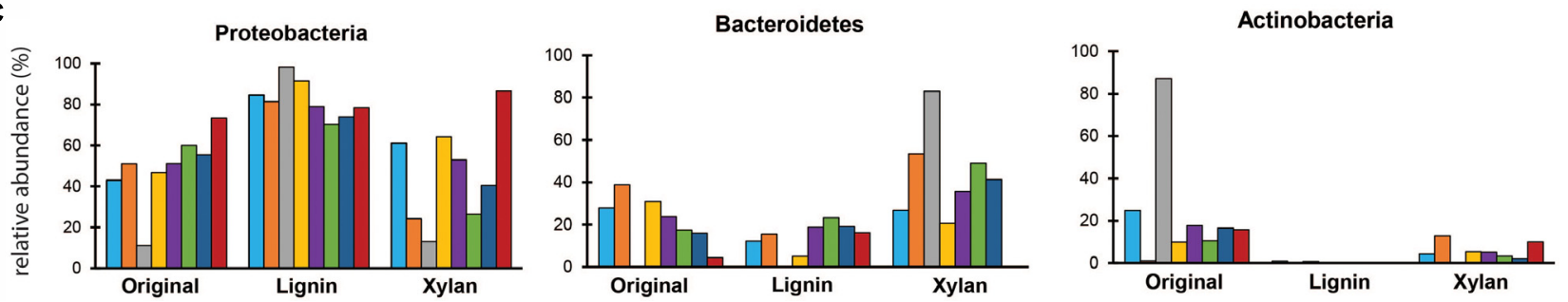

FIGURE 2 | (A) Cluster analysis based on $k$-mer composition dissimilarities among samples $(k=23)$. Colors are used to distinguish enrichment communities. (B) PCoA based on Morisita's overlap index calculated using counts per million reads of Kegg Orthologous on each sample. Oval orange shade was used to highlight the samples after growth in alkali lignin medium. (C) Relative abundance of genes assigned to major bacterial phyla in each sample. Taxonomic assignment was based on the top USEARCH hit.

TABLE 2 | One-way analysis of Morisita's overlap index (ANOSIM) using counts per million reads of Kegg Orthologous.

\begin{tabular}{lcc}
\hline & \multicolumn{2}{c}{ ANOSIM } \\
\cline { 2 - 3 } & $\boldsymbol{R}$ & $\boldsymbol{p}^{*}$ \\
\hline Factor & & \\
Treatment ** & 0.3213 & 0.0002 \\
Enrichment substrate & -0.0432 & 0.6596 \\
Enrichment community & 0.05522 & 0.2245 \\
Inoculum & -0.1016 & 0.9074 \\
Pairwise** & & \\
Original, Lignin & 0.3917 & 0.0006 \\
Original, Xylan & 0.1155 & 0.1059 \\
Lignin, Xylan & 0.4983 & 0.0003 \\
\hline
\end{tabular}

*Bonferroni corrected. **Post hoc analysis. Treatment: Original, Lignin and Xylan. Enrichment substrate: Filter paper and wood. Enrichment community line: C1, C2, C3, S1, S2, S3, S4 and S5. Inoculum: Chicken feces and Soil.

more similar to the ones with the same treatment (Figure 2B), with ones incubated in alkali lignin significantly more similar to each other than to their original communities, though communities after growth xylan medium did not changed significantly from the original communities (Table 2). Factors related to the history of the communities (inoculum, enrichment substrate and the enrichment community line) did not play a significant role on shaping the functional composition of the metagenomes (Table 2).

Taxonomic annotation of genes predicted from our metagenomic data to phylum level revealed that genes belonging to Proteobacteria dominated most of the original consortia, with exception of S3, which was dominated by genes identified as from Actinobacteria (87.1\%) (Figure 2C). After growth in alkali lignin, all the consortia showed an increase in abundance of genes belonging to Proteobacteria, from an average of $48.9 \%$ to an average of $82.14 \%$. When grown in xylan, on average the proportion of Bacteroidetes genes increased from 18.9 to $38.7 \%$, with the exception of the consortia S2_Xyl, S1_Xyl and S4_Xyl (Figure 2C).

More than 270 functions were enriched after growth in alkali lignin (Kruskal-Wallis, corrected $p<0.05$ ) and 399 functions were enriched in the original and/or xylan treatments (Kruskal-Wallis, corrected $p<0.05$ ) (Supplementary Table S3). Functions involved in cellulose degradation were more abundant in the original consortia when compared to the alkali lignin consortia. These functions included cellulose 1,4-beta-cellobiosidases (K19668), endoglucanases (K01179), and beta-glucosidases (K05349 and K05350). Functions assigned to KEGG modules are shown in Figure 3. Genes involved in the catechol ortho-cleavage and benzoate degradation 


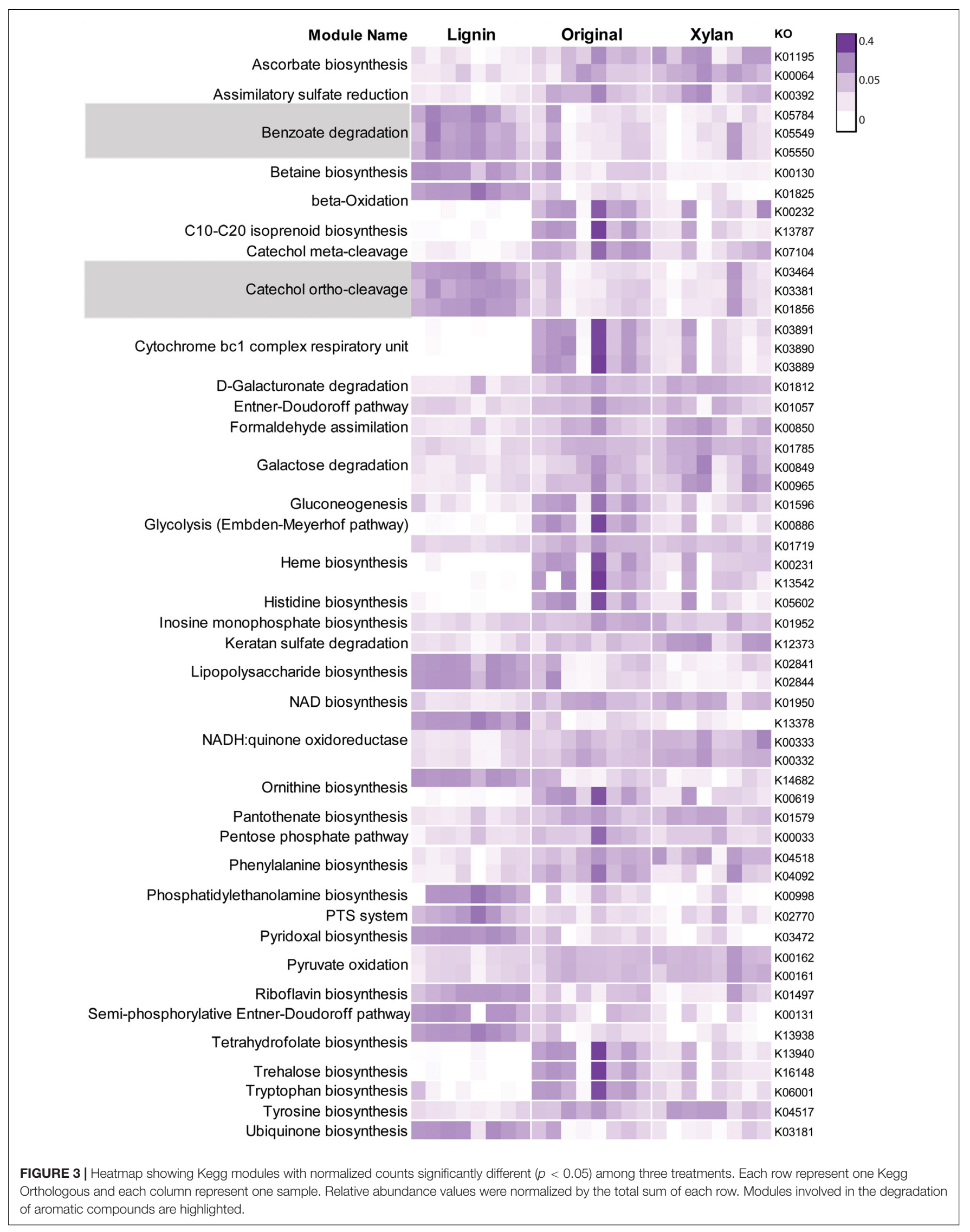




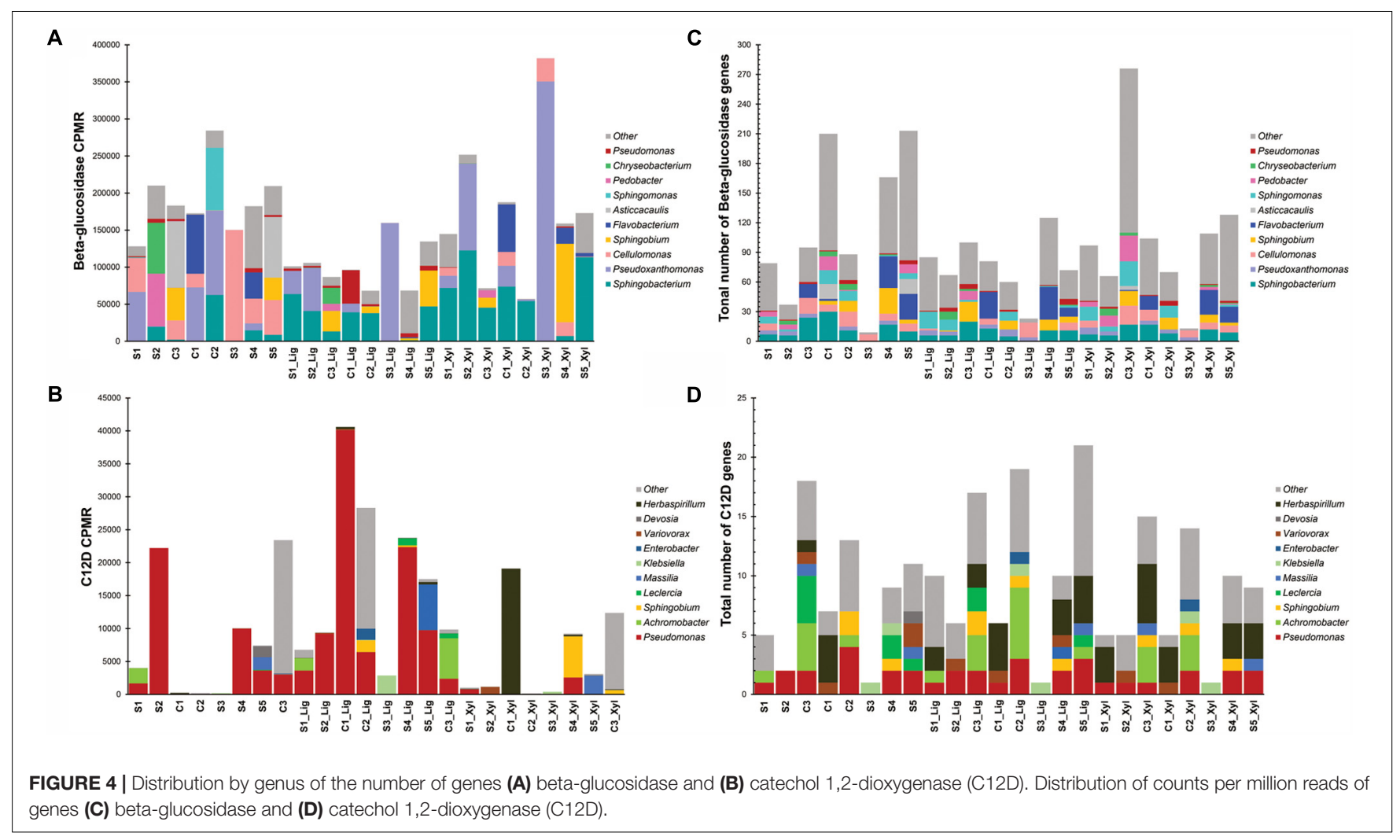

pathways were found to be overrepresented after growth in alkali lignin, while genes involved in pyruvate oxidation and galactose degradation were found overrepresented in the original and xylan samples. Catechol 1,2-dioxygenase (C12D) genes were predominantly classified to the genus Pseudomonas (Figures 4C,D). In contrast, beta-glucosidase genes were primarily classified to the genera Sphingobacterium, Pseudoxanthomonas, and Cellulomonas (Figures 4A,B).

\section{DISCUSSION}

Selective enrichment is a common approach for obtaining lignocellulose-degrading microbial consortia that can be used for biomass conversion to biofuels and other valuable biochemical. Studies have focused on identifying how inoculum sources (Cortes-Tolalpa et al., 2016) and enrichment conditions (de Lima Brossi et al., 2016; Wong et al., 2016) can affect the yield and efficiency of a lignocellulose-degrading microbial consortia. In our study, we aimed to identify community members responsible for the degradation of xylan and ligninderived compounds. We expected that after incubation on xylan and alkali lignin, the resulting communities would be enriched in members that consume each of these substrates, independently of the history of the communities. We observed that after sub-culturing the consortia conserved the overall genetic repertoire of the original consortia, which was assessed by their $k$-mer composition, but the relative abundance of members carrying specific functions changed in response to the carbon source used, as observed with taxonomic and functional annotation.

The functional and taxonomic structure of the communities changed considerably after growth in alkali lignin, both in their taxonomic and functional profiles. Moreover, despite of their enrichment history, most of the consortia converged to a similar composition, with prevalence of Pseudomonas spp. and aromatic degradation genes. As mentioned in the introduction, substratedriven convergence has already been observed in other studies (Jia et al., 2016; Wong et al., 2016). Jia et al. (2016) proposed that community convergence indicates that the selective pressure imposed by the culturing conditions is strong enough to change the initial relative abundance of community members. Lignin-derived compounds are known to be toxic to a various microorganisms, and may provide a competitive advantage to more tolerant species, such as Pseudomonas spp. (Abdelaziz et al., 2016). An increase in the abundance of Pseudomonas was associated with enrichment of genes related to aromatic compound degradation. Lignin is a heterogeneous aromatic polymer, which is found in the plant cell wall covalently crosslinked with polysaccharides (Li M. et al., 2016). Members of the Pseudomonas genus are considered major degraders of aromatic compounds in various environments (Ma et al., 2013; TerrónGonzález et al., 2016) and several studies have demonstrated that the Pseudomonas has the capability to catabolize low molecular weight lignin (Salvachúa et al., 2015; Tian et al., 2016; Mohan and Phale, 2017; Ravi et al., 2017). The strain P. putida CSV86 was shown to utilize aromatics, such as veratryl alcohol and ferulic acid, preferentially over glucose (Basu et al., 2006) via 
the ortho-cleavage pathway (Mohan and Phale, 2017). In our study, Catechol 1,2-dioxygenase (C12D) genes were enriched after growth with alkali lignin as a sole carbon source and were mostly attributed to Pseudomonas (Figure 2). C12D is the key enzyme in the ortho-cleavage pathway and catalyzes the intradiol cleavage of the aromatic ring generating cis,cis-muconic acid (Fuchs et al., 2011) and it is likely involved in the degradation of kraft lignin by Cupriavidus basilensis B-8 (Shi et al., 2013).

On the other hand, after growth in xylan medium, we observed that the shift in taxonomic composition (Figure 2C) was not followed by a significant change of the functional potential of the microbial assemblages (Figure 2B). This can be explained by different substrate preferences of species with similar functional potential, such as Sphingobacterium and Cellulomonas. Sphingobacterium increased after growth in xylan medium and it has been reported in various plant biomassdegrading microbial consortia and was found to secrete endobeta-1,4-xylanases during growth on wheat straw (Jiménez et al., 2015). Cellulomonas strains have also been found to secrete xylanases (Sánchez-Herrera et al., 2007; Lisov et al., 2017). However, in the growth conditions used in our study, Cellulomonas seems to be outcompeted by Sphingobacterium when xylan is the sole carbon source. The original consortia were enriched in genes encoding exoglucanases, endoglucanases, and beta-glucosidases. Three of the original consortia were enriched in filter paper, which is composed of crystalline cellulose, and five consortia were bred in poplar wood chips, which is mainly composed of cellulose. Therefore it suggests that the observed association of Cellulomonas with the original consortia is related to their preference to degrade cellulose (Meinke et al., 1993).

Resource partitioning among distinct bacterial phyla has been demonstrated in soil microbial communities and is a major driver of microbial diversity (Wilson and Lindow, 1994; Goldfarb et al., 2011; Kramer et al., 2016). Our findings provides strong evidence that distinct players in lignocellulolytic microbial consortia have distinct substrate preferences. These bacteria are promising candidates for future studies of engineered microbial consortia in lignocellulose degradation. Studies have observed an increase in degradation rates in mixed cultures, when compared to monocultures (Tiunov and Scheu, 2005; Evans et al., 2017). Identifying key degraders of each plant

\section{REFERENCES}

Abdelaziz, O. Y., Brink, D. P., Prothmann, J., Ravi, K., Sun, M., Garcia-Hidalgo, J., et al. (2016). Biological valorization of low molecular weight lignin. Biotechnol. Adv. 34, 1318-1346. doi: 10.1016/j.biotechadv.2016.10.001

Allgaier, M., Reddy, A. P., Park, J. I., Ivanova, N., D’haeseleer, P., Lowry, S., et al. (2010). Targeted discovery of glycoside hydrolases from a switchgrassadapted compost community. PLoS One 5:e8812. doi: 10.1371/journal.pone.00 08812

Basu, A., Apte, S. K., and Phale, P. S. (2006). Preferential utilization of aromatic compounds over glucose by Pseudomonas putida CSV86. Appl. Environ. Microbiol. 72, 226-2230. doi: 10.1128/AEM.72.3.2226-2230.2006

Ceballos, S. J., Yu, C., Claypool, J. T., Singer, S. W., Simmons, B. A., Thelen, M. P., et al. (2017). Development and characterization of a thermophilic, lignin cell wall polymer is the first step toward the development of engineered microbial consortia for an "optimal" plant biomass degradation. Using metagenomics, we confirmed the major role of Pseudomonas during degradation of lignin-derived aromatic compounds. Additionally, Sphingobacterium is identified as a good candidate for xylan degradation and Cellulomonas seems to play a crucial role during aerobic degradation of cellulose in complexes substrates.

\section{AUTHOR CONTRIBUTIONS}

CC designed and performed the experiments and analyses and also wrote the manuscript. HF performed the bioinformatics analysis and wrote the manuscript. CC designed and wrote the manuscript.

\section{FUNDING}

This work was funded by the U.S. Department of Energy Great Lakes Bioenergy Research Center, Office of Science grant BER DE-FC02-07ER64494.

\section{ACKNOWLEDGMENTS}

We thank Gina R. Lewin and Steven Suh for their work during the establishment of the lignocellulose-adapted communities. We thank the DOE JGI production sequencing and IMG teams for sequencing, assembling, and annotating the metagenomes under the proposal number 503055 - BRC Proposal. We also thank the University of Wisconsin Biotechnology Center DNA Sequence Facility for providing Illumina Next Generation sequencing facilities and services.

\section{SUPPLEMENTARY MATERIAL}

The Supplementary Material for this article can be found online at: https://www.frontiersin.org/articles/10.3389/fmicb. 2018.00364/full\#supplementary-material

degrading microbiota. Process Biochem. 63, 193-203. doi: 10.1016/j.procbio. 2017.08.018

Chen, I. M. A., Markowitz, A. M., Chu, K., Palaniappan, K., Szeto, E., Pillay, M., et al. (2016). IMG/M: integrated genome and metagenome comparative data analysis system. Nucleic Acids Res. 45, D507-D516. doi: 10.1093/nar/gkw929

Cortes-Tolalpa, L., Jiménez, D. J., de Lima Brossi, M. J., Salles, J. F., and van Elsas, J. D. (2016). Different inocula produce distinctive microbial consortia with similar lignocellulose degradation capacity. Appl. Microbiol. Biotechnol. 100, 1256-1263. doi: 10.1007/s00253-016-7516-6

Cragg, S. M., Beckham, G. T., Bruce, N. C., Bugg, T. D. H., Distel, D. L., Dupree, P., et al. (2015). Lignocellulose degradation mechanisms across the Tree of Life. Curr. Opin. Chem. Biol. 29, 108-119. doi: 10.1016/j.cbpa.2015.10.018

de Lima Brossi, M. J., Jiménez, D. J., Cortes-Tolalpa, L., and van Elsas, J. D. (2016). Soil-derived microbial consortia enriched with different plant biomass reveal 
distinct players acting in lignocellulose degradation. Microb. Ecol. 71, 616-627. doi: 10.1007/s00248-015-0683-7

DeAngelis, K. M., Gladden, J. M., Allgaier, M., D'haeseleer, P., Fortney, J. L., Reddy, A., et al. (2010). Strategies for enhancing the effectiveness of metagenomic-based enzyme discovery in lignocellulolytic microbial communities. Bioenergy Res. 3, 146-158. doi: 10.1007/s12155-010-9089-z

Evans, R., Alessi, A. M., Bird, S., McQueen-Mason, S. J., Bruce, N. C., and Bockhurst, M. A. (2017). Defining the functional traits that drive bacterial decomposer community productivity. ISME J. doi: 10.1038/ismej.2017.22

Fan, H., Ives, A. R., Surget-Groba, Y., and Cannon, C. H. (2015). An assembly and alignment-free method of phylogeny reconstruction from next-generation sequencing data. BMC Genomics 16:522. doi: 10.1186/s12864-015-1647-5

Fuchs, G., Boll, M., and Heider, J. (2011). Microbial degradation of aromatic compounds - from one strategy to four. Nat. Rev. Microbiol. 9, 803-816. doi: $10.1038 /$ nrmicro2652

Gessner, M. O., Swan, C. M., Dang, D. K., McKie, B. G., Bardgett, R. D., Wall, D. H., et al. (2010). Diversity meets decomposition. Trends Ecol. Evol. 25, 372-380. doi: $10.1016 /$ j.tree.2010.01.010

Goldemberg, J. (2009). Ethanol for a sustainable energy future. Science 315, 808-810. doi: 10.1126/science.1137013

Goldfarb, K. C., Karaoz, U., Hanson, C. A., Santee, C. A., Bradford, M. A., Treseder, K. K., et al. (2011). Differential growth responses of soil bacterial taxa to carbon substrates of varying chemical recalcitrance. Front. Microbiol. 2:94. doi: 10.3389/fmicb.2011.00094

Haruta, S., Cui, Z., Huang, Z., Li, M., Ishii, M., and Igarashi, Y. (2002). Construction of a stable microbial community with high cellulose-degradation ability. Appl. Microbiol. Biotechnol. 59, 529-534. doi: 10.1007/s00253-002$1026-4$

Huntemann, M., Ivanova, N. N., Mavromatis, K., Tripp, H. J., Paez-Espino, D., Tennessen, K., et al. (2016). The standard operating procedure of the DOEJGI metagenome annotation pipeline (MAP v.4). Stand. Genomic. Sci. 11:17. doi: 10.1186/s40793-016-0138-x

Jia, Y., Wilkins, D., Lu, H., Cai, M., and Lee, P. K. H. (2016). Long-term enrichment on cellulose or xylan causes functional and taxonomic convergence of microbial communities from anaerobic digesters. Appl. Environ. Microbiol. 82, 1519-1529. doi: 10.1128/AEM.03360-15

Jiménez, D. J., Brossi, M. J. L., Schückel, J., Kračun, S. K., Willats, W. G. T., and van Elsas, J. D. (2016). Characterization of three plant biomass-degrading microbial consortia by metagenomics- and metasecretomics-based approaches. Appl. Microbiol. Biotechnol. 100, 10463-10477. doi: 10.1007/s00253-0167713-3

Jiménez, D. J., Dini-Andreote, F., DeAngelis, K. M., Singer, S. W., Salles, J. F., and van Elsas, J. D. (2017). Ecological insights into the dynamics of plant biomassdegrading microbial consortia. Trends Microbiol. 5, 788-796. doi: 10.1016/j.tim. 2017.05.012

Jiménez, D. J., Maruthamuthu, M., and van Elsas, J. D. (2015). Metasecretome analysis of a lignocellulolytic microbial consortium grown on wheat straw, xylan and xylose. Biotechnol. Biofuels 8:199. doi: 10.1186/s13068-0150387-8

Kato, S., Chino, K., Kamimura, N., Masai, E., Yumoto, I., and Kamagata, Y. (2015). Methanogenic degradation of lignin-derived monoaromatic compounds by microbial enrichments from rice paddy field soil. Sci. Rep. 5:14295. doi: 10.1038/ srep14295

Klein-Marcuschamer, D., and Blanch, H. W. (2015). Renewable fuels from biomass: technical hurdles and economic assessment of biological routes. AlChE J. 61, 2689-2701. doi: 10.1002/aic. 14755

Korenblum, E., Jiménez, D. J., and van Elsas, J. D. (2016). Succession of lignocellulolytic bacterial consortia bred anaerobically from lake sediment. Microb. Biotechnol. 9, 224-234. doi: 10.1111/1751-7915.12338

Kramer, S., Dibbern, D., Moll, J., Huenninghaus, M., Koller, R., and Krueger, D. (2016). Resource partitioning between bacteria, fungi, and protists in the detritusphere of an agricultural Soil. Front. Microbiol. 7:1524. doi: 10.3389/ fmicb.2016.01524

Kurtz, S., Phillippy, A., Delcher, A. L., Smoot, M., Shumway, M., Antonescu, C., et al. (2004). Versatile and open software for comparing large genomes. Genome. Biol. 5:R12.

Lemos, L. N., Pereira, R. V., Quaggio, R. B., Martins, L. F., Moura, L. M. S., da Silva, A. R., et al. (2017). Genome-centric analysis of a thermophilic and cellulolytic bacterial consortium derived from composting. Front. Microbiol. 8:644. doi: $10.3389 /$ fmicb.2017.00644

Lewin, G. R., Johnson, A. L., Soto, R. D. M., Perry, K., Book, A. J., Horn, H. A., et al. (2016). Cellulose-enriched microbial communities from leafcutter ant (Atta colombica) refuse dumps vary in taxonomic composition and degradation ability. PLoS One 11:e0151840. doi: 10.1371/journal.pone.01 51840

Li, D., Luo, R., Liu, C. M., Leung, C. M., Ting, H. F., Sadakane, K., et al. (2016). MEGAHIT v1.0: A fast and scalable metagenome assembler driven by advanced methodologies and community practices. Methods 102, 3-11. doi: 10.1016/j. ymeth.2016.02.020

Li, M., Pu, Y., and Ragauskas, A. J. (2016). Current understanding of the correlation of lignin structure with biomass recalcitrance. Front. Chem. 4:15. doi: 10.3389/ fchem.2016.00045

Liao, J. C., Mi, L., Pontrelli, S., and Luo, S. (2016). Fuelling the future: microbial engineering for the production of sustainable biofuels. Nat. Rev. Microbiol. 14, 288-304. doi: 10.1038/nrmicro.2016.32

Lisov, A. V., Belova, O. V., Lisova, Z. A., Vinokurova, N. G., Nagel, A. S., Andreeva-Kovalevskaya, Z. I., et al. (2017). Xylanases of Cellulomonas flavigena: expression, biochemical characterization, and biotechnological potential. $A M B$ Express 7:5. doi: 10.1186/s13568-016-0308-7

Ma, J., Xu, L., and Jia, L. (2013). Characterization of pyrene degradation by Pseudomonas sp. strain Jpyr-1 isolated from active sewage sludge. Bioresour. Technol. 140, 15-21. doi: 10.1016/j.biortech.2013.03.184

Markowitz, V. M., Chen, I. A., Palaniappan, K., Chu, K., Szeto, E., Pillay, M., et al. (2013). IMG 4 version of the integrated microbial genomes comparative analysis system. Nucleic Acids Res. 42, D560-D567. doi: 10.1093/nar/ gkt963

Maruthamuthu, M., Jiménez, D. J., Stevens, P., and van Elsas, J. D. (2016). A multi-substrate approach for functional metagenomics-based screening for (hemi)cellulases in two wheat straw-degrading microbial consortia unveils novel thermoalkaliphilic enzymes. BMC Genomics 17:86. doi: 10.1186/s12864016-2404-0

Meinke, A., Gilkes, N. R., Kilburn, D. G., Miller, R. C. Jr., and Warren, R. A. (1993). Cellulose-binding polypeptides from Cellulomonas fimi: endoglucanase D (CenD), a family A beta-1,4-glucanase. J. Bacteriol. 175, 1910-1918. doi: $10.1128 / \mathrm{jb}$.175.7.1910-1918.1993

Mello, B. L., Alessie, A. M., McQueen-Mason, S., Bruce, N. C., and Polikarpov, I. (2016). Nutrient availability shapes the microbial community structure in sugarcane bagasse compost-derived consortia. Sci. Rep. 6:38781. doi: 10.1038/ srep38781

Mohan, K., and Phale, P. S. (2017). Carbon source-dependent inducible metabolism of veratryl alcohol and ferulic acid in Pseudomonas putida CSV86. Appl. Environ. Microbiol. 31:e3326-16. doi: 10.1128/AEM.03326-16

Mosier, N., Wyman, C., Dale, B., Elander, R., Lee, Y. Y., Holtzapple, M., et al. (2005). Features of promising technologies for pretreatment of lignocellulosic biomass. Bioresour. Technol. 96, 373-386. doi: 10.1016/j.biortech.2004. 06.025

Pritchard, L., Glover, R. H., Humphris, S., Elphinstone, J. G., and Toth, I. K. (2016). Genomics and taxonomy in diagnostics for food security: softrotting enterobacterial plant pathogens. Anal. Methods 8, 12-24. doi: 10.1039/ C5AY02550H

Ravi, K., García-Hidalgo, J., Gorwa-Grauslund, M. F., and Lidén, G. (2017). Conversion of lignin model compounds by Pseudomonas putida KT2440 and isolates from compost. Appl. Microbiol. Biotechnol doi: 10.1007/s00253-0178211-y

Salvachúa, D., Karp, E. M., Nimlos, C. T., Vardon, D. R., and Beckham, G. T. (2015). Towards lignin consolidated bioprocessing: simultaneous lignin depolymerization and product generation by bacteria. Green Chem. 17, 4951-4967. doi: 10.1039/C5GC01165E

Sánchez-Herrera, L. M., Ramos-Valdivia, A. C., de la Torre, M., Salgado, L. M. and Ponce-Noyola, T. (2007). Differential expression of cellulases and xylanases by Cellulomonas flavigena grown on different carbon sources. Appl. Microbiol. Biotechnol. 77, 589-595. doi: 10.1007/s00253-007-1190-7

Shi, I., Chai, L., Tang, C., Yang, Z., Zhang, H., Chen, R., et al. (2013). Characterization and genomic analysis of kraft lignin biodegradation by the beta-proteobacterium Cupriavidus basilensis B-8. Biotechnol. Biofuels 6:1. doi: 10.1186/1754-6834-6-1 
Terrón-González, L., Martín-Cabello, G., Ferrer, M., and Santero, E. (2016). Functional metagenomics of a biostimulated petroleum-contaminated soil reveals an extraordinary diversity of extradiol dioxygenases. Appl. Environ. Microbiol. 82, 8-12. doi: 10.1128/AEM.03811-15

Tian, J. H., Pourcher, A. M., and Peu, P. (2016). Isolation of bacterial strains able to metabolize lignin and lignin-related compounds. Lett. Appl. Microbiol. 63, 30-37. doi: 10.1111/lam.12581

Tiunov, A. V., and Scheu, S. (2005). Facilitative interactions rather than resource partitioning drive diversity-functioning relationships in laboratory fungal communities. Ecol. Lett. 8, 618-625. doi: 10.1111/j.1461-0248.2005. 00757.x

Voelskow, H. (1988). Extrazelluläre mikrobielle polysaccharide: polymere mit interessanten anwendungsmöglichkeiten. Forum Mikrobiol. 6, 273-283.

Wang, Y., Liu, Q., Yan, L., Gao, Y., Wang, Y., and Wang, W. (2013). A novel lignin degradation bacterial consortium for efficient pulping. Bioresour. Technol. 139, 113-113. doi: 10.1016/j.biortech.2013.04.033

Wei, H., Xu, Q., Taylor, L. E. II, Baker, J. O., Tucker, M. P., and Ding, S. H. (2009). Natural paradigms of plant cell wall degradation. Curr. Opin. Biotechol. 20, 330-338. doi: 10.1016/j.copbio.2009.05.008
Wilson, M., and Lindow, S. E. (1994). Coexistence among epiphytic bacterial populations mediated through nutritional resource partitioning. Appl. Environ. Microbiol. 60, 4468-4477.

Wong, M. T., Wang, W., Lacourt, M., Couturier, M., Edwards, E. A., and Master, E. R. (2016). Substrate-driven convergence of the microbial community in lignocellulose-amended enrichments of gut microflora from the Canadian Beaver (Castor canadensis) and North American Moose (Alces americanus). Front. Microbiol. 7:961. doi: 10.3389/fmicb.2016.00961

Conflict of Interest Statement: The authors declare that the research was conducted in the absence of any commercial or financial relationships that could be construed as a potential conflict of interest.

Copyright (c) 2018 Carlos, Fan and Currie. This is an open-access article distributed under the terms of the Creative Commons Attribution License (CC BY). The use, distribution or reproduction in other forums is permitted, provided the original author(s) and the copyright owner are credited and that the original publication in this journal is cited, in accordance with accepted academic practice. No use distribution or reproduction is permitted which does not comply with these terms. 